\title{
Estudio descriptivo de la patología observada en una consulta de cirugía de mano
}

\author{
I. Proubasta Renart ${ }^{(1)}$, I. Gich Saladrich ${ }^{(2)}$ \\ Hospital de SANT Pau. Barcelona \\ (1) Servicio de Cirugía Ortopédica y Traumatología \\ (2) SERVICIO DE FARMACIA
}

\author{
Correspondencia: \\ Dr. Ignasi Proubasta Renart \\ Servicio de Cirugía Ortopédica y Traumatología \\ Hospital de la Santa Cruz y San Pablo \\ C/ Mas Casanovas, 90 \\ 08025 Barcelona \\ e-mail: iproubasta@santpau.es
}

Objetivo: Analizar mediante un estudio observacional descriptivo, la frecuencia de las patologías observadas en una consulta de cirugía de mano.

Material y método: Estudio observacional descriptivo, de una serie consecutiva de 400 pacientes visitados en una consulta de cirugía de mano, analizando estadísticamente la edad, género y patología observada.

Resultados: Nueve patologías (artrosis trapecio metacarpiana, enfermedad de Dupuytren, síndrome del túnel carpiano, dedo en resorte, epicondilalgia, artrosis de las interfalángicas distales, enfermedad de De Quervain, ganglión de muñeca y neuropatía cubital), abarcaron el $75 \%$ de todos los pacientes visitados, y el resto (25\%), correspondieron a otras 29 patologías diferentes. Del total de pacientes, 237 $(59.25 \%)$ correspondieron al género femenino, con una edad media de 56 años ( $\mathrm{r}=16-88$; DE: 16.89), y $163(40.75 \%)$ al masculino, con una edad media de 48,95 años ( $r=17-79$; DE: 17.86).

Conclusiones: $\mathrm{Si}$ en el $75 \%$ de los pacientes visitados en una consulta de cirugía de mano, el grueso de las patologías observadas se circunscriben a nueve, es posible incidir en ellas para que la enseñanza de nuestros residentes y médicos en general sea la más adecuada.

Palabras clave: Cirugía de Mano, Recursos, Bases de datos clínico-administrativos.
Purpose: To analyze by means of a descriptive observational study, the frequency of the pathologies observed in a clinic hand unit

Material and method: Study observational and descriptive, of a consecutive series of 400 patients visited in a consultation of clinic hand unit, analyzing statistically the age, gender and observed pathology.

Results: Nine pathologies (trapeciometacarpal joint arthrosis, Dupuytren disease, carpal tunnel syndrome, trigger finger, epycondilitis, distal interphalangeal joint arthrosis, DeQuervain tendynitis, ganglion of the wrist and ulnar neuropathy), included $75 \%$ of all the visited patients, and the rest $(25 \%)$, corresponded to other 29 different pathologies. Of the total of patients, $237(59,25 \%)$ they were female, with an average age of 56 years $(\mathrm{r}=16-88$; OF: 16.89), and $163(40,75 \%)$ they were male, with an average age of 48.95 years $(r=17-79$; OF: 17.86).

Conclusions: If in $75 \%$ of the patients visited in a clinic hand unit, the thickness of the observed pathologies is confined to nine, is possible to affect them so that the education of our residents and doctors, in general, is adapted.

Key words: Hand Surgery, Trends, Clinical-administrative data bases. 


\section{INTRODUCCIÓN}

$\mathrm{E}$ n España, no existe información médica referente a la demanda y recursos en cirugía de mano. El propósito del presente trabajo fue el de averiguar dichos parámetros en nuestro medio, con el fin de poder responder convenientemente a las necesidades asistenciales de nuestra población, así como adecuar un programa de formación específico en cirugía de mano para los médicos internos residentes (MIR) en los hospitales universitarios.

\section{MATERIAL Y MÉTODO}

De forma prospectiva, se analizaron consecutivamente 400 pacientes adultos que fueron atendidos por primera vez en la Unidad de Cirugía de Mano del Servicio de Cirugía Ortopédica y Traumatología del Hospital de la Santa Cruz y San Pablo de Barcelona (España) durante el año 2008. Se trata de un hospital universitario de tercer nivel perteneciente a la red hospitalaria de utilización pública del Sistema Catalán de la Salud, con 630 camas y 34 especialidades, y con un área de influencia de 300.000 habitantes. Se excluyeron del estudio todos los casos traumáticos y pediátricos; los primeros por ser visitados, diagnosticados y tratados inicialmente en el Servicio de Urgencias, y los segundos por ser un grupo muy concreto de pacientes que eran atendidos exclusivamente en la Unidad de Cirugía Pediátrica Ortopédica.

Los parámetros valorados fueron la edad, género y patología observada, y en cuanto al tratamiento estadístico, las variables categóricas fueron descritas facilitando el número de casos y su porcentaje, mientras que para las variables cuantitativas, se calcularon los valores medio, mínimo y máximo, así como la desviación estándar.

\section{RESULTADOS}

\section{- Edad y género:}

Del total de los 400 pacientes visitados, 237 $(59.25 \%)$ correspondieron al género femenino, con una edad media de 56 años $(\mathrm{r}=16-88$; DE: 16.89), y 163 (40.75\%) al masculino, con una edad media de 48,95 años ( $\mathrm{r}=17-79$; DE: 17.86).

\section{TABLA I - NúMERO Y PORCENTAJES DE LAS 9 PATOLOGÍAS MÁS FRECUENTES OBSERVADAS EN UN DISPENSARIO DE CIRUGÍA DE MANO}

\begin{tabular}{|l|c|c|}
\hline & $\mathbf{N}^{\circ}$ casos & $\%$ \\
\hline 1) Artrosis trapeciometacarpiana & 66 & 16.5 \\
\hline 2) Enfermedad de Dupuytren & 56 & 14.0 \\
\hline 3) Síndrome del túnel carpiano & 44 & 11.0 \\
\hline 4) Dedo en resorte & 42 & 10.5 \\
\hline 5) Epicondilalgia & 30 & 7.5 \\
\hline 6) Artrosis interfalángicas distales & 16 & 4.0 \\
\hline 7) Enfermedad de De Quervain & 16 & 4.0 \\
\hline 8) Ganglión de muñeca & 16 & 4.0 \\
\hline 9) Neuropatía cubital & 14 & 3.5 \\
\hline Total & 300 & 75.0 \\
\hline
\end{tabular}




\section{TABLA II - NúMERO Y PORCENTAJES DE LAS DIFERENTES PATOLOGíAS OBSERVADAS EN UNA} CONSUlta de CiRugía de MANO, EXCLUYENDO LAS 9 MÁs FRECUENTES (TABLA I)

\begin{tabular}{|c|c|c|}
\hline & $\mathbf{N}^{\circ}$ casos & $\%$ \\
\hline 1) Artrosis metacarpofalángica & 7 & 1.75 \\
\hline 2) Artrosis radiocubital distal & 6 & 1.5 \\
\hline 3) Rotura subcutánea extensor largo pulgar & 6 & 1.5 \\
\hline 4) Epitroclealgia & 5 & 1.25 \\
\hline 5) Artrosis interfalángicas proximales & 5 & 1.25 \\
\hline 6) Quiste mucoide & 5 & 1.25 \\
\hline 7) Secuelas sección tendones flexores & 5 & 1.25 \\
\hline 8) Síndrome de intersección & 4 & 1 \\
\hline 9) Mano reumática & 4 & 1 \\
\hline 10) Tendinitis palmar mayor & 4 & 1 \\
\hline 11) Tumores mano & 4 & 1 \\
\hline 12) Tumor de células gigantes vainas tendinosas & 4 & 1 \\
\hline 13) Secuelas fracturas radio distal & 4 & 1 \\
\hline 14) Inestabilidad metacarpofalángica del pulgar & 4 & 1 \\
\hline 15) Disociación escafosemilunar & 3 & 0.75 \\
\hline 16) Quiste vaina flexora & 3 & 0.75 \\
\hline 17) Deformidad en ojal & 3 & 0.75 \\
\hline 18) Síndrome doloroso regional complejo & 3 & 0.75 \\
\hline 19) Muñeca SLAC & 3 & 0.75 \\
\hline 20) Muñeca SNAC & 3 & 0.75 \\
\hline 21) Lesión fibrocartílago triangular & 3 & 0.75 \\
\hline 22) Tumor glómico dedo & 2 & 0.5 \\
\hline 23) Artrosis radiocarpiana & 2 & 0.5 \\
\hline 24) Tofos gotosos mano & 2 & 0.5 \\
\hline 25) Enfermedad de Kienböck & 2 & 0.5 \\
\hline 26) Secuelas lesión rama motora N. Mediano & 1 & 0.25 \\
\hline 27) Secuelas sección nervios colaterales dedos & 1 & 0.25 \\
\hline 28) Deformidad en cuello de cisne postraumática & 1 & 0.25 \\
\hline 29) Carpo giboso & 1 & 0.25 \\
\hline Total & 100 & 25 \\
\hline
\end{tabular}


Tabla III - Relación Edad/patología, en las nueve patologías más FRECUENTES OBSERVADAS EN UNA CONSULTA DE CIRUGÍA DE MANO

\begin{tabular}{lcccc} 
& \multicolumn{4}{c}{ Edad (años) } \\
\cline { 2 - 5 } Patología & Media & DE & mín & máx \\
\hline Artrosis trapeciometacarpiana & 63,9 & 7,8 & 52 & 80 \\
Artrosis interfalángica distal & 63,8 & 5,9 & 57 & 70 \\
Síndrome túnel carpiano & 60,9 & 20,3 & 33 & 90 \\
Dedo en resorte & 60,6 & 8,5 & 51 & 77 \\
Enfermedad de Dupuytren & 60,1 & 15,4 & 42 & 81 \\
Enfermedad de De Quervain & 54,6 & 14,3 & 32 & 73 \\
Neuropatía cubital & 52,8 & 12,2 & 41 & 80 \\
Epicondilalgia & 49,5 & 14 & 28 & 75 \\
Ganglión & 31,7 & 11,4 & 16 & 53 \\
\hline
\end{tabular}

DE: desviación estándar.

\section{- Patologías observadas:}

Se observó que 9 de ellas constituían el grueso de la serie y, dentro de éstas, solo cuatro superaban el $10 \%$ de los casos (Tabla I). Estas cuatro patologías fueron: la artrosis trapecio metacarpiana (16,5\%), enfermedad de Dupuytren (14\%), síndrome del túnel carpiano $(11 \%)$ y dedo en resorte $(10.5 \%)$, sumando el $52 \%$ de todos los casos, mientras que las otras cinco siguientes patologías más frecuentes, aunque ninguna superó el 10\%, fueron: epicondilalgia (7.5\%), artrosis de las interfalángicas distales (4\%), enfermedad de De Quervain $(4 \%)$, ganglión de muñeca (4\%) y neuropatía cubital (3.5\%), las cuales sumaron el $23 \%$ de toda la serie. En definitiva, las nueve patologías comentadas abarcaron el $75 \%$ de todos los pacientes visitados y el resto (25\%), correspondieron a otras 29 patologías diferentes (Tabla II).

\section{- Relación edad/ patología observada:}

En este apartado solo se estudió dicha relación en las 9 patologías más frecuentes (Tabla III), observando que la edad media de los pacientes varió entre los 49,5 y 63,9 años.

\section{DISCUSIÓN}

Los resultados de este estudio permiten conocer la frecuencia de las patologías más comu- nes observadas en un dispensario de cirugía de mano de un hospital universitario español. En este sentido, 9 patologías específicas (Tabla I) fueron diagnosticadas en el $75 \%$ de los pacientes, mientras que en el $25 \%$ restante se diagnosticaron otras 29 (Tabla II). Si comparamos nuestros resultados absolutos con los de otros estudios de las mismas características ${ }^{1-3}$, observamos que son muy parecidos en cuanto a las patologías observadas, pero no tanto en cuanto a los datos demográficos, es decir, edad y género (Tabla III). Dicho hallazgo podría explicarse por el área de influencia de nuestro hospital, la cual abarca un distrito de la ciudad donde existe una población de mayor edad, especialmente mujeres. De hecho, si nos fijamos en la tabla III, nuestros pacientes tenían una edad entre los 49,5 y 63,9 años, mientras que en el estudio llevado a cabo por Burke et al. ${ }^{2}$ en 1990, los pacientes con el mismo grupo de patologías se situó entre los 40,8 y los 61.3 años. Sin embargo, en el estudio de Crouch et al. ${ }^{1}$, la población observada osciló entre los 35 y 55 años de edad, cifra inferior a las dos precedentes, aunque hay que mencionar que en la misma se incluyeron todos los casos traumáticos, patologías éstas que suelen afectar más a pacientes jóvenes. Por lo que respecta al género, en nuestra serie el femenino ocupó el $60 \%$ de los casos, mientras que en el estudio de Crouch et al. ${ }^{1}$, la proporción entre hombres y mujeres fue de 2 a 1. Sin embargo, también 
aquí, hemos de hacer mención que las patologías traumáticas suelen afectar más a hombres que a mujeres.

En cuanto a la patologías observadas, creemos que las mismas no difieren con el paso del tiempo, tal como observó en el estudio llevado a cabo por Wildin et al. ${ }^{3}$, en el que compararon dos grupos de pacientes separados en el tiempo por una década (1989-1990 y 2000-2001) y en el que objetivaron que la prevalencia de las patologías de una comunidad no se alteran, al menos durante un periodo de 10 años. A destacar en nuestra serie que, tanto la artrosis trapeciometacarpiana, como el síndrome del túnel carpiano, enfermedad de Dupuytren, dedo en resorte y la artrosis interfalángica distal, afectó a personas entre los 60,1 y los 63,9 años. Este hecho, per se, podría explicar la elevada frecuencia con la que se da la asociación entre varias de las patologías citadas, es decir, pacientes con artrosis trapecimetacarpiana y síndrome del túnel carpiano, síndrome del túnel carpiano y enfermedad de Dupuytren, o artrosis simultánea de la articulaciones trapecimetacarpiana e interfalángicas distales.

Aún siendo importantes los parámetros demográficos, es indudable que existen una serie de patologías propias de la mano que presentan una frecuencia elevada en la población y que, en nuestra serie se circunscribieron a nueve, lo que supuso el $75 \%$ de los pacientes visitados en un año, cifra suficientemente significativa a tener en cuenta a la hora de enfocar la enseñanza de nuestros residentes y médicos en general.

\section{BIBLIOGRAFÍA}

1. Crouch CC, O'Connor DP, Pierce $P$, et al. Utilization of orthopaedic services for hand and wrist conditions in a capitated population. J Bone Joint Surg A, 2004; 86: 51-6.
2. Burke FD, Dias JJ, Lunn MJ, et al. Providing care hand disorders - trauma and elective. $\mathrm{J}$ Hand Surg B, 1991; 16: 13-8.
3. Wildin C, Dias JJ, Heras-Palou $\mathrm{C}$, et al. Trends in elective hand surgery referrals from primare care. Ann R Coll Surg Engl, 2006; 88: 543-6. 